\title{
DISTRIBUTED PARAMETER SYSTEMS WITH A MULTIPLICATIVE FRACTIONAL GAUSSIAN NOISE ${ }^{1}$
}

\author{
T. E. Duncan * B. Pasik-Duncan ** \\ * Department of Mathematics, University of Kansas, Lawrence, \\ KS 66045, duncan@math. ku.edu \\ ** Department of Mathematics, University of Kansas, Lawrence, \\ KS 66045, bozenna@math.ku.edu
}

\begin{abstract}
A fractional Gaussian noise is used in a stochastic differential equation in a Hilbert space to model a stochastic distributed parameter system. An explicit solution is given for this stochastic differential equation. Copyright ${ }^{\complement} 2005$ IFAC
\end{abstract}

Keywords: Distributed parameter systems, Fractional Brownian motion, Stochastic systems

\section{INTRODUCTION}

Distributed parameter systems provide models for partial differential equations and time delay differential equations. Often these systems are subject to perturbations or the systems have errors in the parameters either of which are effectively modeled by stochastic processes that can be described as a multiplicative Gaussian noise. Fractional Brownian motion is a family of Gaussian processes that are indexed by the Hurst parameters $H \in(0,1)$. These processes have been noted empirically in a wide variety of physical phenomena especially for $H \in(1 / 2,1)$. Fractional Brownian motions with values in $\mathbb{R}^{n}$ were introduced by Kolmogorov (1940) and some important properties of these processes were given by Mandelbrot and Van Ness (1968). In this paper, the formal derivative of a (cylindrcal) fractional Brownian motion in a Hilbert space with $H \in(1 / 2,1)$ is used to describe a multiplicative Gaussian noise in a distributed parameter systems. This fractional Gaussian noise is used in a stochastic differential equation in a Hilbert space to model a stochastic distributed parameter system. An explicit solution is given for this stochastic differential equation.

\footnotetext{
1 Research supported in part by NSF Grants DMS 0204669 and ANI 0125410.
}

Since a fractional Brownian motion for $H \in(1 / 2,1)$ is not a semimartingale, a stochastic calculus, that is different from the one for Brownian motion, is required (Alòs and Nualart, 2003; Duncan et al., 2000; Duncan et al., 2005b). Some features of this stochastic calculus (Duncan et al., 2005b) are noted. There are only limited results for the solutions of stochastic differential equations even in finite dimensional spaces. An explicit solution for a distributed parameter system with a finite dimensional multiplicative fractional Gaussian noise is given in (Duncan et al., 2005a). It seems that there are no other results for solutions of these models with a multiplicative Gaussian noise.

\section{PRELIMINARIES AND MAIN RESULT}

Initially a standard cylindrical fractional Browinian motion in a separable Hilbert space is defined and some aspects of a stochastic calculus for such a process are reviewed.

Definition 1. Let $V$ be a separable Hilbert space with inner product $\langle\cdot, \cdot\rangle$. A continuous, zero mean, cylindrical Gaussian process in $V\left(B^{H}(t), t \geq 0\right)$ on the complete probability space $(\Omega, \mathcal{F}, P)$ is said to be a standard cylindrical fractional Brownian motion with 
the Hurst parameter $H \in(0,1)$ if $\mathbb{E}\left[\left\langle\psi, B^{H}(t)\right\rangle\right]=0$ for all $\in \mathbb{R}$ and $\psi \in V$ and

$$
\begin{aligned}
& \mathbb{E}\left[\left\langle\psi_{1}, B^{H}(s)\right\rangle\left\langle\psi_{2}, B^{H}(t)\right\rangle\right] \\
& \quad=\left\langle\psi_{1}, \psi_{2}\right\rangle \frac{1}{2}\left[t^{2 H}+s^{2 H}-|t-s|^{2 H}\right]
\end{aligned}
$$

for all $s, t \in \mathbb{R}_{+}$and $\psi_{1}, \psi_{2} \in V$.

A family of stochastic processes is introduced.

Definition 2. Let $H \in(1 / 2,1)$. The linear space $L_{H}^{2}\left([0,1], \mathcal{L}_{2}(U, V)\right)$, often denoted by $L_{H}^{2}$, where $\mathcal{L}_{2}(U, V)$ is the family of Hilbert-Schmidt operators from $U$ to $V$, is the family of $\mathcal{L}_{2}(U, V)$-valued generalized processes on $(\Omega, \mathcal{F}, P)$ such that $X \in L_{H}^{2}$ if

(i) the map

$$
(s, \omega) \mapsto\left\langle X^{\prime}(s, \omega) \psi, \varphi\right\rangle_{U}
$$

is $\mathcal{B}([0,1]) \otimes \mathcal{F}$ measurable for all $\varphi \in U$, $\psi \in V$.

$$
\text { (ii) } \begin{aligned}
|X|_{L_{H}^{2}}^{2} & =\mathbb{E} \int_{0}^{1}\left(u_{1 / 2-H}(s)\right. \\
& \left.<\infty, \quad \times\left|I_{1-}^{H-1 / 2}\left(u_{H-1 / 2} X\right)(s)\right|_{\mathcal{L}_{2}}\right)^{2} d s
\end{aligned}
$$

where $I_{1-}^{H-1 / 2}$ is a fractional integral (Samko et al., 1993).

Remark. The norm of $L_{H}^{2}$ can often be expressed in terms of $\phi_{H}=H(2 H-1)|\cdot|{ }^{2 H-2}$, so that $L_{H}^{2}$ is the completion of the pre-Hilbert space of (uniformly bounded) processes $(X(t), t \in[0,1])$ with the following inner product

$$
\mathbb{E} \int_{0}^{1} \int_{0}^{1}\langle X(s), X(t)\rangle_{\mathcal{L}_{2}} \phi_{H}(s-t) d s d t .
$$

It is convenient to introduce a family of elementary random variables that are used in the construction of a stochastic integral.

Definition 3. The linear space $\mathcal{S}$ is the family of smooth, cylindrical, $V$-valued random variables on $(\Omega, \mathcal{F}, P)$ such that if $F \in \mathcal{S}$, then it has the form

$$
F=\sum_{j=1}^{n} f_{j}\left(\int_{0}^{1} \gamma_{1 j} d B^{H}, \ldots, \int_{0}^{1} \gamma_{n_{j} j} d B^{H}\right) \eta_{j}
$$

where $\eta_{j} \in V, \gamma_{k j} \in L_{\phi_{H}}^{2}\left([0,1], \mathcal{L}_{2}(U, \mathbb{R})\right), f_{j} \in$ $C_{p}^{\infty}\left(\mathbb{R}^{n_{j}}\right)$ for $j \in\{1, \ldots, n\}$ and $k \in\left\{1, \ldots, n_{j}\right\}$ and $C_{p}^{\infty}\left(\mathbb{R}^{n}\right)$ is the set of $f: \mathbb{R}^{n} \rightarrow \mathbb{R}$ where $f \in$ $C^{\infty}$ and $f$ and all of its derivatives have polynomial growth.

Definition 4. The derivative $D: \mathcal{S} \rightarrow L_{H}^{2}$ is a linear operator which is given for $F \in \mathcal{S}$ in Definition 3 by

$D_{t} F=\sum_{j=1}^{n} \sum_{i=1}^{n_{j}} \frac{\partial f_{j}}{\partial x_{i}}$

$$
\begin{aligned}
& \times\left(\int_{0}^{1} \gamma_{1 j} d B^{H}, \ldots, \int_{0}^{1} \gamma_{n_{j} j} d B^{H}\right) \\
& \times \eta_{j} \otimes \gamma_{i j}(t) .
\end{aligned}
$$

The stochastic integral is defined as a dual to $D$.

Definition 5. Let $X \in L_{H}^{2}$. The $\mathcal{L}_{2}(U, V)$-valued generalized process $X$ is integrable with respect to $B^{H}$ if $F \mapsto\langle X, D F\rangle_{L_{H}^{2}}$ is continuous on $\mathcal{S}$ with the $L^{2}(\Omega)$ norm topology. The stochastic integral $\int_{0}^{1} X d B^{H}$ is a zero mean, $V$-valued random variable such that

$$
\langle X, D F\rangle_{L_{H}^{2}}=\mathbb{E}\left\langle\int_{0}^{1} X d B^{H}, F\right\rangle_{V}
$$

for each $F \in \mathcal{S}$.

Let $D_{H}^{1,2}=\operatorname{Dom}(D)$, so that $D: D_{H}^{1,2} \rightarrow L_{H}^{2}$. Now, a family of processes is defined that are integrable according to the Definition 5 so that the stochastic integral is defined.

Definition 6. Let $H \in(1 / 2,1)$. The space

$$
L_{H}^{1,2}\left([0,1], \mathcal{L}_{2}\left(U, D_{H}^{1,2}\right)\right)
$$

or simply $L_{H}^{1,2}$ is the family of $\mathcal{L}_{2}\left(U, D_{H}^{1,2}\right)$-valued generalized processes $(X(t), t \in[0,1])$ on $(\Omega, \mathcal{F}, P)$ such that

i) $X:[0,1] \times \Omega \rightarrow \mathcal{L}_{2}\left(U, D_{H}^{1,2}\right)$ is $\mathcal{B}([0,1]) \otimes$ $\mathcal{F}$ measurable,

ii) There is a measurable version of $\left(D_{s} X(t)\right.$, $s, t \in[0,1])$, that is, the map $(s, t, \omega) \mapsto$ $\left\langle\left(D_{s} X(t, \omega)\right) \varphi, \psi\right\rangle$ is $\mathcal{B}\left([0,1]^{2}\right) \otimes \mathcal{F}$ measurable for each $X$ and all $\varphi \in U$ and $\psi \in V$, and

iii)

$$
\begin{aligned}
&|X|_{L_{H}^{1,2}}^{2}=\langle X, X\rangle_{L_{H}^{1,2}} \\
&=\mathbb{E} \int_{0}^{1}\left\langle u_{1 / 2-H}(s) I_{1-}^{H-1 / 2}\left(u_{H-1 / 2} X\right)(s),\right. \\
&\left.u_{1 / 2-H}(s) I_{1-}^{H-1 / 2}\left(u_{H-1 / 2} X\right)(s)\right\rangle_{\mathcal{L}_{2}} \\
& \times d s \\
&+\mathbb{E} \int_{0}^{1} \int_{0}^{1}\left\langle u_{1 / 2-H}(s) u_{1 / 2-H}(t) I_{1-}^{H-1 / 2}\right. \\
& \times\left(u_{H-1 / 2}(q) I_{1-}^{H-1 / 2}\right. \\
& \times\left(u_{H-1 / 2}(r)\right. \\
&\left.\left.\times D_{r} X(q)\right)(s)\right)(t), \\
& \quad(t) I_{1-}^{H-1 / 2} \\
& \times\left(u_{H-1 / 2}(q) I_{1-}^{H-1 / 2}\right. \\
& \times\left(u_{H-1 / 2}(r)\right. \\
&\left.\left.\left.\times D_{r} X(q)\right)(s)\right)(t)\right\rangle_{\mathcal{L}_{2}} \\
& \times d s d t<\infty .
\end{aligned}
$$


The space $L_{H}^{1,2}$ is a Hilbert space with the inner product induced from the norm. It is the completion of the pre-Hilbert space of uniformly bounded $\mathcal{L}_{2}(U, \mathcal{S})$ valued processes with the inner product

$$
\begin{aligned}
\langle X, X\rangle_{L_{H}^{2}} & \\
+\mathbb{E} \int_{0}^{1} \int_{0}^{1} \int_{0}^{1} \int_{0}^{1} & \left\langle D_{p} X(q), D_{r} X(s)\right\rangle_{\mathcal{L}_{2}} \\
& \times \phi_{H}(p-s) \phi_{H}(r-q) \\
& \times d p d q d r d s
\end{aligned}
$$

For notational convenience there is some abuse of the notation $|\cdot|_{\mathcal{L}_{2}}$ and $\langle\cdot, \cdot\rangle_{\mathcal{L}_{2}}$ because these can refer to different families of Hilbert-Schmidt operators. However, the appropriate family of operators should be clear from the context.

The following result verifies that the processes in $L_{H}^{1,2}$ are integrable and $L_{H}^{1,2}$ is a natural family of integrands because the stochastic integral satisfies an isometry.

Theorem 1. If $X \in L_{H}^{1,2}\left([0,1], \mathcal{L}_{2}\left(U, D_{H}^{1,2}\right)\right)$, then $X$ is integrable with respect to $B^{H}$, so the stochastic integral $\int_{0}^{1} X d B^{H}$ is a well defined zero mean $V$ valued random variable in $L^{2}(\Omega)$. Furthermore, if $X, Y \in L_{H}^{1,2}$, then

$$
\mathbb{E}\left\langle\int_{0}^{1} X d B^{H}, \int_{0}^{1} Y d B^{H}\right\rangle_{V}=\langle X, Y\rangle_{L_{H}^{1,2}} .
$$

In applications of stochastic integration, a change of variables or Itô formula is especially useful. An Itô formula is given here for a smooth function of a $V$ valued process that is defined by an equation that contains a stochastic integral of a standard cylindrical fractional Brownian motion.

Let $(X(t), 0 \leq t \leq 1)$ be a $V$-valued process with continuous sample paths that satisfies the stochastic equation

$$
X(t)=X(0)+\int_{0}^{t} a(r) d r+\int_{0}^{t} b(r) d B^{H}(r) \quad \text { a.s. }
$$

for $t \in[0,1]$, where $X(0) \in V$ is deterministic, the stochastic integral is defined by Definition 5 and the $V$-valued process $(a(t), 0 \leq t \leq 1)$ and the $\mathcal{L}_{2}(U, V)$-valued process $(b(t), 0 \leq t \leq 1)$ are $\sigma\left(B^{H}(t), 0 \leq t \leq 1\right)$ measurable and furthermore satisfy

$$
\begin{aligned}
\mathbb{E}\left[\int_{0}^{1} \int_{0}^{1} \int_{0}^{q}|a(p)|_{V}^{2} d p \phi_{H}(q-r) d r d q\right. \\
\left.\quad+\int_{0}^{1} \int_{0}^{1} \int_{0}^{q}\left|D_{r} a(p)\right|_{\mathcal{L}_{2}}^{2} d p \phi_{H}(r-q) d r d q\right] \\
\quad<\infty
\end{aligned}
$$

and

$$
\mathbb{E}\left[\int_{0}^{1} \int_{0}^{1}|b(q)|_{\mathcal{L}_{2}}^{2} \phi_{H}(r-q) d r d q\right.
$$

$$
\begin{aligned}
& +\int_{0}^{1} \int_{0}^{1} \int_{0}^{1} \int_{0}^{1}\left|D_{r} b(p)\right|_{\mathcal{L}_{2}}^{2} \phi_{H}(p-t) \\
& \times \phi_{H}(r-q) d p d q d r d t \\
& +\int_{0}^{1} \int_{0}^{1} \int_{0}^{1} \int_{0}^{1} \int_{0}^{1} \int_{0}^{1}\left|D_{\alpha} D_{r} b(p)\right|_{\mathcal{L}_{2}}^{2} \\
& \times \phi_{H}(r-t) \\
& \times \phi_{H}(\beta-p) \\
& \times \phi_{H}(\alpha-q) \\
& \times d \alpha d \beta d p d q d r d t] \\
& <\infty \text {. }
\end{aligned}
$$

Theorem 2. Let $(X(t), 0 \leq t \leq 1)$ be the process given above that satisfies the associated integrability assumptions above. Let $F: V \rightarrow V$ be a twice continuously differentiable function with $F^{\prime}$ and $F^{\prime \prime}$ uniformly bounded in the operator norms. Then the process $(F(X(t)), 0 \leq t \leq 1)$ satisfies the stochastic equation

$$
\begin{aligned}
& F(X(t)) \\
&= F(X(0))+\int_{0}^{t} F^{\prime}(X(r)) a(r) d r \\
&+\int_{0}^{t} F^{\prime}(X(r)) b(r) d B^{H}(r) \\
&+\int_{0}^{t} \int_{0}^{1} \int_{0}^{p} \operatorname{tr}_{U} F^{\prime \prime}(X(p))\left(D_{q} a(r), b(p)\right) \\
&+\int_{0}^{t} \int_{0}^{1} \int_{0}^{p} \operatorname{tr}_{U} F^{\prime \prime}(X-q) d r d q d p \\
& \times\left(\left(D_{q} b(r)\right) d B^{H}(r), b(p)\right) \\
& \times \phi_{H}(p-q) d q d p
\end{aligned}
$$

where $F^{\prime}$ and $F^{\prime \prime}$ are the first and the second derivatives respectively.

These stochastic calculus results are proved in (Duncan et al., 2005b).

A (strong) solution is explicitly given to a stochastic differential equation in a Hilbert space that can be used to model some stochastic partial differential equations (cf. (Duncan et al., 2005a) for some examples). The stochastic differential equation is one with a multiplicative fractional Gaussian noise that is given by

$$
\begin{aligned}
d X(t) & =A X(t) d t+\sum_{n=1}^{\infty} C_{n} X(t)\left\langle l_{n}, d B^{H}(t)\right\rangle \\
X(0) & =x_{0}
\end{aligned}
$$

where $x_{0}, X(t) \in V, H \in(1 / 2,1),\left(l_{n}, n \in \mathbb{N}\right)$ is a complete, orthonormal basis of $V$ and $\left(C_{n}, n \in \mathbb{N}\right)$ is an $\mathcal{L}(V)$-valued sequence. 
The following assumptions are used to obtain a solution.

(A1) The linear operator $A$ with domain $\operatorname{Dom}(A)$ generates a strongly continuous semigroup $(T(t)$, $t \geq 0)$.

(A2) The family of linear operators $\left(A, C_{n}, n \in \mathbb{N}\right)$ is a commuting family on $D(A)$ and

$$
\sum_{n=1}^{\infty}\left|C_{n}\right|^{2}<\infty \text {. }
$$

Definition 7. A strong solution of the equation (1) is a $\mathcal{B}([0,1]) \otimes \mathcal{F}$ measurable and $D(A)$-valued process $(X(t), t \in[0,1])$ such that

$$
P\left(\int_{0}^{1}(|X(s)|+|A X(s)|) d s<\infty\right)=1,
$$

the $V$-valued process $\left(\sum_{n=1}^{\infty} C_{n} X(t), t \in[0,1]\right)$ satisfies a.s. Definition 6 (iii) without the expectation so that the stochastic integral in (1) is well defined and for each $t \in[0,1]$

$$
\begin{aligned}
X(t)= & x_{0}+\int_{0}^{t} A X(s) d s \\
& +\int_{0}^{t} \sum_{n=1}^{\infty} C_{n} X(s)\left\langle l_{n}, d B^{H}(s)\right\rangle \quad \text { a.s. }
\end{aligned}
$$

The following main result gives an explicit expression for a strong solution of (1).

Proposition 1. If (A1) and (A2) are satisfied and $x_{0} \in$ $\operatorname{Dom}(A)$, then there is a strong solution of $(1)$ that is given by

$$
\begin{aligned}
X(t)= & \exp \left[\sum_{n=1}^{\infty} C_{n}\left\langle l_{n}, B^{H}(t)\right\rangle-H t^{2 H-1} \sum_{n=1}^{\infty} C_{n}^{2}\right] \\
& \times T(t) x_{0} .
\end{aligned}
$$

A proof of this result is given in (Duncan et al., $2005 b$ ). The proof is a direct application of the Itô formula in Theorem 2 given there.

These stochastic differential equations can be used to model stochastic partial differential equations with a multiplicative noise. Some examples are described in (Duncan et al., 2005a).

\section{REFERENCES}

Alòs, Elisa and David Nualart (2003). Stochastic integration with respect to the fractional Brownian motion. Stoch. Stoch. Rep. 75(3), 129-152.

Duncan, T. E., B. Maslowski and B. Pasik-Duncan (2005a). Stochastic equations in Hilbert space with a multiplicative fractional Gaussian noise. to appear.

Duncan, T. E., J. Jakubowski and B. Pasik-Duncan $(2005 b)$. Stochastic integration for fraction Brownian motion in a Hilbert space. preprint.
Duncan, Tyrone E., Yaozhong Hu and Bozenna PasikDuncan (2000). Stochastic calculus for fractional Brownian motion. I. Theory. SIAM J. Control Optim. 38(2), 582-612 (electronic).

Kolmogorov, A. N. (1940). Wienersche Spiralen und einige andere interessante Kurven im Hilbertschen Raum. C. R. (Doklady) Acad. Sci. URSS (N.S.) 26, 115-118.

Mandelbrot, Benoit B. and John W. Van Ness (1968). Fractional Brownian motions, fractional noises and applications. SIAM Rev. 10, 422-437.

Samko, Stefan, Anatoly Kilbas and Oleg Marichev (1993). Fractional integrals and derivatives. Gordon and Breach Science Publishers. Yverdon. Theory and applications, Edited and with a foreword by S. M. Nikol'skiǔ, Translated from the 1987 Russian original, Revised by the authors. 